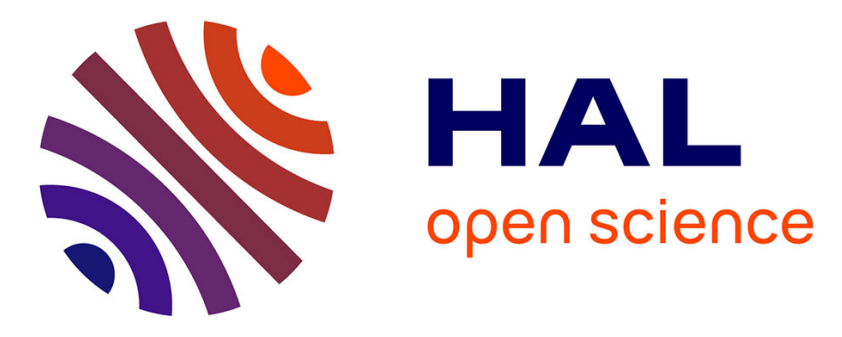

\title{
Virtual hybrid test control of sinuous crack
}

Clément Jailin, Andreea Carpiuc, Kyrylo Kazymyrenko, Martin Poncelet, Hugo Leclerc, François Hild, Stéphane Roux

\section{To cite this version:}

Clément Jailin, Andreea Carpiuc, Kyrylo Kazymyrenko, Martin Poncelet, Hugo Leclerc, et al.. Virtual hybrid test control of sinuous crack. Journal of the Mechanics and Physics of Solids, 2017, 102, pp.239 - 256. 10.1016/j.jmps.2017.03.001 . hal-01549224

\section{HAL Id: hal-01549224 \\ https://hal.science/hal-01549224}

Submitted on 28 Jun 2017

HAL is a multi-disciplinary open access archive for the deposit and dissemination of scientific research documents, whether they are published or not. The documents may come from teaching and research institutions in France or abroad, or from public or private research centers.
L'archive ouverte pluridisciplinaire HAL, est destinée au dépôt et à la diffusion de documents scientifiques de niveau recherche, publiés ou non, émanant des établissements d'enseignement et de recherche français ou étrangers, des laboratoires publics ou privés. 


\title{
Virtual hybrid test control of sinuous crack
}

\author{
Clément Jailin ${ }^{\mathrm{a}}$, Andreea Carpiuc ${ }^{\mathrm{a}, \mathrm{b}}$, Kyrylo Kazymyrenko ${ }^{\mathrm{b}}$, Martin \\ Poncelet $^{\mathrm{a}}$, Hugo Leclerc ${ }^{\mathrm{a}}$, François Hild ${ }^{\mathrm{a}}$, Stéphane Roux ${ }^{\mathrm{a}}$ \\ ${ }^{a}$ Laboratoire de Mécanique et Technologie (LMT) \\ ENS Paris-Saclay/CNRS/Université Paris-Saclay \\ 61 avenue du Président Wilson, 94235 Cachan, France \\ ${ }^{b}$ Institut des Sciences de la Mécanique et Applications Industrielles \\ UMR EDF-CNRS-CEA-ENSTA 9219, Université Paris-Saclay \\ 828 Boulevard des Maréchaux, 91762 Palaiseau Cedex, France
}

\begin{abstract}
The present study aims at proposing a new generation of experimental protocol for analysing crack propagation in quasi brittle materials. The boundary conditions are controlled in real-time to conform to a predefined crack path. Servo-control is achieved through a full-field measurement technique to determine the pre-set fracture path and a simple predictor model based on linear elastic fracture mechanics to prescribe the boundary conditions on the fly so that the actual crack path follows at best the predefined trajectory. The final goal is to identify, for instance, non-local damage models involving internal lengths. The validation of this novel procedure is performed via a virtual testcase based on an enriched damage model with an internal length scale, a prior chosen sinusoidal crack path and a concrete sample. Notwithstanding the fact that the predictor model selected for monitoring the test is a highly simplified picture of the targeted constitutive law, the proposed protocol exhibits a much improved sensitivity to the sought parameters such as internal lengths as assessed from the comparison with other available experimental tests.

Keywords: A. Crack propagation and arrest. B. Concrete. Crack mechanics.
\end{abstract} Sensors and actuators. C. Finite element.

2016 MSC: 00-01, 99-00 


\section{Introduction}

Mechanical and civil engineering simulations are based on increasingly complex constitutive models. They enable the behaviour of materials and real structures to be predicted with better accuracy at the expense of more demanding

5 calibration steps. On the one hand, such models have the potential to reduce the need for very expensive large scale tests in service conditions. On the other hand, calibration of the constitutive parameters and assessment of their domain of validity becomes more challenging and requires either new tests (potentially at small scale), or new procedures to exploit at best a richer information. Enhanced sensitivity to model parameters thus becomes a crucial issue in designing optimal experimental tests.

For quasi brittle materials such as concrete, there are different ways of modelling the development of cracking. Smeared crack models were first introduced [1, 2]. Continuum damage mechanics is an alternative route with local [3] 15 and non-local formulations $4,5,6,7,8,9$. Cohesive zone models were also proposed to handle initiation and propagation in such materials [10, 11, 12]. In crack band models, the finite width of the process zone is accounted for [13]. Variational formulations can also describe these two different stages of propagation [14. Discrete descriptions based on lattice calculations are considered to 20 account for multiple cracking [15].

All these models need to be calibrated with possibly discriminating experiments. Stress/strain data are the typical information for calibrating smeared crack and local damage models [16]. For non-local damage models, load/deflection curves of so-called size effect tests can be used [17. The width 25 of the process zone was shown to be very useful for tuning such models [18]. Point measurements were used by these authors. The process zone width can be revealed via digital image correlation (DIC) [19]. Similarly, the roughness of the fractured surface was also measured and considered for calibration purposes [9]. Conversely, many models only require ultimate strength properties to be known 30 to get first order estimations of their parameters (e.g., see Refs. [8, 20]). From 
this analysis it is concluded that very few studies use full-field measurements for identification and validation purposes. DIC was used to monitor complex experiments 21] and calibrate a cohesive zone model 22]. The aim of the present work is to propose such a procedure via virtual testing that explicitly considers full-field measurements and their uncertainties.

A recent series of crack propagation tests on concrete samples [21] has been performed to achieve complex and discriminating crack patterns (and partly used in an on-going benchmark [23]) by modifying the loading path during the experiment. The mechanical loading was prescribed on the top and bottom part of the sample by a hexapod [24, 25, 26] (see Figure 1) and the crack tip position was obtained thanks to 2D DIC [27, 28]. First, the results of these tests show that it is possible to manually control the stability and the deflection of a crack in a quasi brittle material using plane loading, namely, a combination of tension, shear and in-plane rotation. Then it has been shown that these complex cracks with many changes of propagation direction and branching were sensitive to parameters of a state-of-the-art non-local damage model [20].
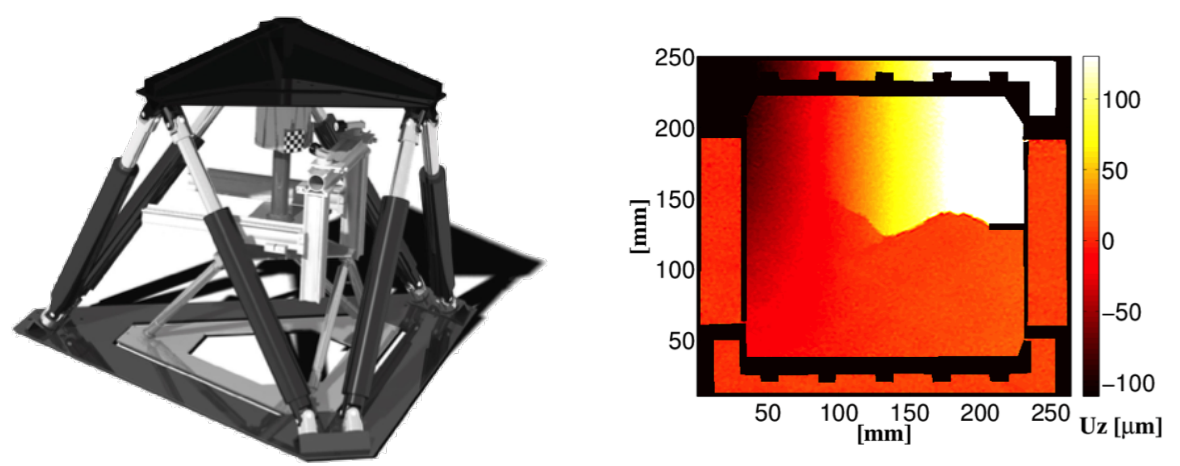

Figure 1: (a) Multiaxial testing machine (hexapod) with six degrees of freedom and (b) vertical displacement field on a concrete sample measured via DIC after having manually monitored the loading so that the crack path successively propagates along predetermined directions [21]

Manual control of the loading path in a multiaxial testing machine to achieve such crack propagation experiment is thus possible, but extremely delicate and definitely non-optimal. Automated load control would be highly desirable. It 
would allow for a much more reactive feedback on loading directions and magnitudes, hence leading to an enhanced accuracy in following a predetermined crack path, and provide the needed flexibility to reveal parameters that are difficult to calibrate. This, undoubtedly, would pave the way to 'smart' and adaptive experiments, a kind of holy Grail in optimised mechanical tests.

The design of such control loops is challenging. The first generation of mechanical tests appeared in the late eighteenth century, which were based on a load defined before performing the experiment. The second generation of tests followed when they were controlled by a local and measurable quantity of interest (e.g., strain). This was also true for multiaxial experiments [29, 30, 31. Such experimental possibilities now flourish up to a full 3D control, which with 6 degrees of freedom becomes very demanding in terms of servo-control [24, 25, 26]. In spite of this remarkable progress, none of these types of experiments meets the above objective. It is the authors' belief that the control of experiments via numerical simulations (e.g., finite element computations) updated in realtime with full-field measurements constitutes a major breakthrough and open new perspectives. As such they can be seen as a sort of '3rd generation experiments.' It also enters the framework of dynamic data-driven application systems 32] whose emergence is one critical aspect of simulation-based engineering sciences [33]. Therefore, an interaction between the mechanical loading, 70 the measurements and the model must be considered, thereby defining the basis of a hybrid testing procedure (Figure 2).

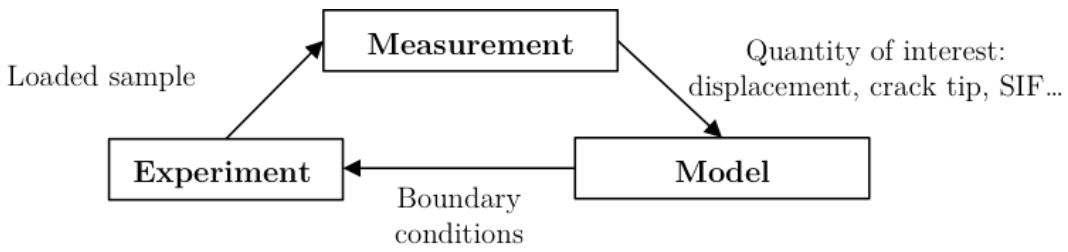

Figure 2: Principle of 3rd generation tests

In the literature, most hybrid experiments reported so far are the so-called 
'sub-structured hybrid tests' 34, 35, 36, 37. The latter ones address the dynamic response of generally large-scale structures, and are currently a very active and promising field of research. The spirit of the method is to split the structure into at least two parts. Among them, those that have a simple mechanical behaviour are computationally simulated and referred to as numerical sub-domains (or substructures). The other parts, which are critical and complex (e.g., involving non-linearities, irreversibility), the so-called physical sub-domains, are kept as substructures actually tested in the laboratory. Pseudo-dynamic testing, continuous pseudo-dynamic testing, fast hybrid testing, real-time substructure testing and real-time dynamic substructure testing [38, 39, 36, 40] are methodologies developed within this hybrid framework and where part of the structure is substituted by computations. On the one hand, these hybrid tests can greatly reduce the overall cost since most of the structure is simulated numerically, while the much smaller key elements are studied experimentally. Moreover this enhanced cost-effectiveness involves no prejudice to the realism of the mechanically tested part. On the other hand, the models used for the numerical sub-domains are to be well-mastered and simple enough so that computations can be performed quickly to perform dynamic tests. In addition, the measurement devices used for feedback are generally poor and frequently reduced to few local strain gauges or LVDTs and often no more than one single actuator is used. These limitations are nothing but the manifestations of fairly recent and novel developments. The main objective is to focus the experiment on a substructure, and substitute the environment - expected to be simple and well-understood — by a model and controlled actuators.

In contrast, although the proposed hybrid approach shares a number of common features and tools, it does not rely on a partition of the structure into parts. In the above-mentioned desired monitoring, the tested and simulated structure or material specimen are shared, and perfectly superimposed. Hence, an additional challenge to be faced is that the behaviour is not yet fully determined at the stage where control is to take action.

The proposed strategy is to use a simple predictor model - a.k.a. 'low- 
level' model — that is able to predict (to first-order accuracy) crack propagation and orientation. During the experiment, the quantities of interest (e.g., crack tip, local displacement, stress intensity factor) are measured on the specimen and then compared to the set-point values, and the error (i.e., the difference between the prescribed and current variables) is introduced in the 'low-level' model that enables the loading condition to be readjusted in order to follow the pre-established target. The use of a simple model is desirable because it allows for fast computations as needed for real-time control. However, it is legitimate to wonder whether driving the experiment with a model that is different from the one to be identified, does not simply impede the sought identification. It will be argued that, on the contrary, exploiting the discrepancies between designed and achieved crack path, stress intensity factor, or loading history will reveal extremely powerful.

This method allows, for example, a test to be controlled by quantities of interest that are not directly accessible (e.g., crack tip, stress intensity factors, damage fields) but need numerical simulations to be run. The principle of this innovative testing procedure is to establish a continuous (and possibly seamless) communication between the experiment and the numerical simulation. By numerically solving an inverse problem, the boundary conditions are computed on the fly to obtain the sought history (i.e., crack path in the present case). Being able to modify the boundary conditions as functions of the actual behaviour of the specimen enables the loading history to be adapted during the experiment in order to better control the quantity of interest. This procedure may seem similar to traditional tests, but here the choice of quantity of interest becomes nearly unlimited, thereby opening new possibilities for more discriminating tests.

To perform these so-called 3rd generation innovative tests, the hybrid loop is composed of three main parts:

- An experimental loading device (e.g., hexapod) applying multiaxial loading histories to samples enabling cracks to propagate in stable increments along various directions. 
- A measurement technique (e.g., DIC, Integrated-DIC) to determine the position of the crack tip [41, 42, 43] and injecting it in a control algorithm.

- A control algorithm, which is based on a mechanical model, adapting the boundary conditions in order to follow a predefined crack path via numerical simulations.

For obvious security and set-up design reasons, a first step toward a real 3rd generation experiment is the development of a 'virtual' test. The present paper discusses such method to control simulated quasi static plane crack propagation on a quasi brittle sample and its validation with an identification procedure on a test-case. A 'high-level' model thus numerically simulates crack propagation that is considered as a representation of 'true' fracture and substitutes a real experimental set-up. Then the crack tip is numerically detected on the simulated experiment and is used in the 'low-level' model that will adapt the loading to follow the crack path, which is pre-established (i.e., before the experiment). The virtual test is an important contribution to the development and the optimization of the control technique. Equally important is the assessment, from the virtual test, of the feasibility of various crack paths and their figures of merit for identification purposes.

In the present study, a sinusoidal crack path is chosen as the target. The motivations for this choice are numerous:

- changes in propagation directions bring a wealth of information that a straight path cannot reveal, such as mode mixity, damage ahead of the crack tip, or other physical microscale mechanisms affecting crack propagation may be masked by symmetry,

- the periodicity of the sinusoidal shape allows the repeatability of the experiment to be probed, at least approximatively,

- the mean (large scale) crack direction remains straight and hence the path can be kept distant from boundaries, 
- the amplitude and wavelength of the path can be tuned to optimise the test.

The paper is organised as follows. First, the general methodology and com-

\section{Control Principle of the hybrid test}

In this section the content of control part of the hybrid loop (see Figure 3), which can be used for real or virtual hybrid tests, is described.

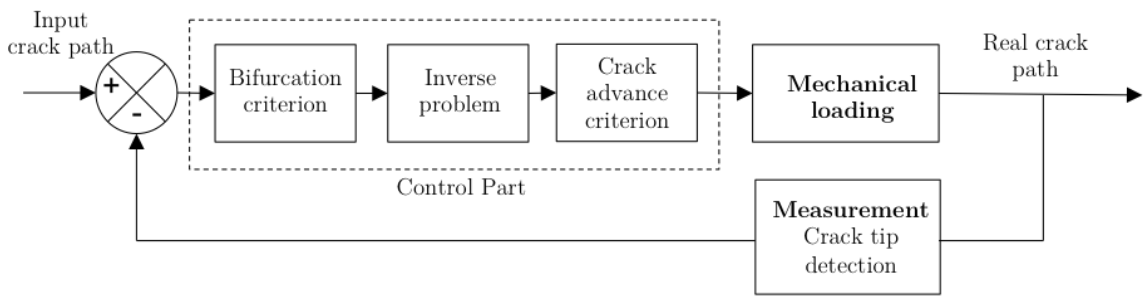

Figure 3: Block diagram of 3rd generation tests

The possible experimental setup is not specified in this work since it could be rather generic, provided that it possesses two important features. First, boundary conditions have to be applied to the specimen using at least two independent degrees of freedom, which influence crack propagation in different ways (otherwise propagation and orientation will not be controlled independently). Second, the measurement of the crack tip position should be possible during crack propagation (otherwise the error calculation will not be possible). For 
be a suitable candidate to conduct such an experiment [21. The crack tip measurement may rely on Integrated Digital Image Correlation (I-DIC) using, for example, Williams' series [44, 45, 42, or any other suitable technique.

For a virtual test, the experiment is replaced by a finite element (FE) simulation of crack propagation. In the present case, a non-local damage model is used. The crack tip position is obtained by a damage field analysis, but any other numerical procedure based on, say, FE results could also be considered. The control part is used to find the boundary conditions to obtain the a priori chosen crack path, which is established before running the test. It consists of three steps: (1) a deflection angle has to be determined and linked to the stress intensity factors (SIFs), (2) these SIFs have to be converted into boundary conditions, (3) a criterion, which controls the advance of the crack per step, has to be satisfied.

This inverse problem and the evaluation of the SIFs are computed using the 'low-level' model, which is in the present case a Linear Elastic Fracture Mechanics (LEFM) analysis coupled with the geometry updating procedure provided by the eXtended Finite Element Method (X-FEM) [46, 47, 48,

\subsection{Crack deflection angle}

During mixed-mode propagation, the crack may move away from the chosen path. To correct the path by applying a new loading a deflection angle criterion has to be used. (It is worth noting that this method will not be applicable if the process zone is larger than the crack path curvature, because the crack will not be influenced by the short-term fluctuation of the loading). Let us consider a given crack path and the measured crack propagation, which is simulated by the 'high-level' (i.e., damage) model. In the same spirit as in the field of robotics and automation for a robot following a path [4], a 'look-ahead distance' is chosen. A first idea (Figure 4 (a)) is to look for the intersection between the chosen (input) crack path and a circle of radius $d r$, which can be considered as a path discretization parameter. This intersection becomes the next target point 
target.

For sufficiently large values of $d r$ it is impossible to describe the full crack path due to its curvature. The shorter $d r$, the more precise the final crack surface. However, if during the experiment, the 'real' crack path happens to be out of $d r$ circle the procedure may fail. Moreover the smaller $d r$, the more sensitive to measurement uncertainty, material heterogeneities and propagation fluctuations. Special attention should be paid while choosing $d r$, namely, smaller than the radius of curvature of the crack path but larger than the process zone.

To address the previous issue, another simpler method is chosen herein. Because the crack propagation component along the $y$-axis is small compared with the component along $x$ (i.e., the crack propagates mainly in the $x$-direction), the circle can be replaced by a straight line parallel to the $y$-axis, with an offset of $d x \approx d r$. The deflection angle is thus chosen as the angle to reach the intersection between the input crack and the vertical line (see Figure 4 (b)). The advantage of this simplified procedure is that the algorithm does not fail, even though the determined crack orientation angle is restricted to cracks whose propagation never recedes along the $x$-axis.

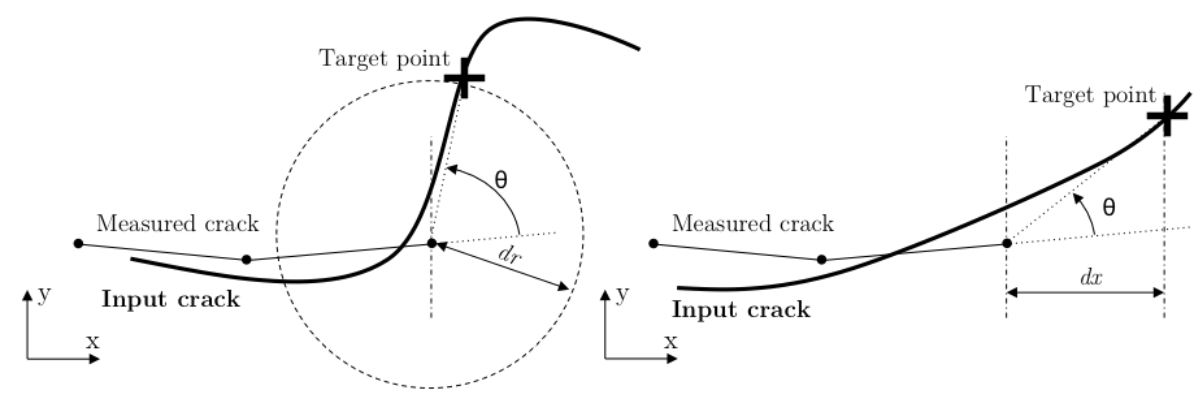

(a)

(b)

Figure 4: Target point with the two proposed methods, (a) at a distance $d r$ from the previous measured point, and (b) at a projected distance $d x$ on the $x$-axis

With the chosen procedure and $d r$ set, the predetermined crack path may not be reached if the propagation step is shorter than $d r$ or if the deflection angle reaches its maximum threshold value. Consequently, the crack will follow 
in the best case a path that will be called the command crack path, namely, the discretised version of the initial input path.

\subsection{Inverse problem}

In the previous section, an algorithm has been proposed, which allows the

Many methods were developed in the past for crack stability control, starting from the simple SIF gradient or principal stresses analysis, going to more complex force-displacement snap-back identification to finally end up with highly non-linear Rayleigh quotient minimization problem. As a first approximation we limit ourselves to linear elasticity and hence introduce the stability criterion 
as some general linear constraint on three in-plane motion amplitudes. The advantage of such description is that the whole relationships remain linear and read as a $3 \times 3$ system $(\{\boldsymbol{k}\}=[\boldsymbol{S}]\{\boldsymbol{b}\})$

$$
\left\{\begin{array}{c}
K_{I} \\
K_{I I} \\
\kappa
\end{array}\right\}=\left[\begin{array}{ccc}
S_{I}^{1} & S_{I}^{2} & S_{I}^{3} \\
S_{I I}^{1} & S_{I I}^{2} & S_{I I}^{3} \\
S_{\kappa}^{1} & S_{\kappa}^{2} & S_{\kappa}^{3}
\end{array}\right] \quad\left\{\begin{array}{l}
b_{1} \\
b_{2} \\
b_{3}
\end{array}\right\}
$$

where $K_{I}, K_{I I}$ are the total SIFs that control crack propagation, $S_{I}^{i}$ and $S_{I I}^{i}$ the sensitivity matrix elements, i.e., SIFs generated by elementary boundary loadings $b_{i}$ (e.g., kinematic (translation and rotation), static (force and torque) components), and $S_{\kappa}^{i}$ a set of parameters that defines the stabilising motion constraint

$$
\kappa=S_{\kappa}^{1} b_{1}+S_{\kappa}^{2} b_{2}+S_{\kappa}^{3} b_{3}
$$

where $S_{\kappa}^{i}$ parameters could be either set during the whole experiment or may vary depending on the crack tip position. The easy way to establish all sensitivity matrix elements $S_{I, I I}^{i}$ is to perform numerical simulations to assess the influence of each elementary loading on the SIFs. To summarise, in order to inverse and solve this system (Equation (1)), three main ingredients are needed, namely, deflection, arrest and stability criteria:

- Deflection criterion. Within the LEFM framework it is commonly assumed that, first, the SIF amplitude sets the crack propagation threshold, and second, the SIF ratio $K_{I I} / K_{I}$ influences the crack deflection angle. While many different deflection criteria exist (e.g., maximum normal stress criterion [50], local symmetry criterion [51, 52]), in order to simplify the analysis the deflection angle is given by an implicit function of SIF ratio

$$
f(\theta)=\frac{K_{I I}}{K_{I}}
$$

- Arrest criterion. With the ratio of load amplitude $b_{i}, i=1-3$, set by the deflection angle and $K_{I}$ gradient, a proportional loading is applied until the crack increment length reaches $d r$. The advantage of this iterative 
methodology is that it is directly controlled by the measured quantity of interest, i.e., the crack length. In another class of possible strategies (e.g., prediction method) the missing equation needed to get the boundary conditions should be provided. For example, the boundary amplitude may be chosen to satisfy the LEFM propagation criterion $K_{I}=K_{I c}$. One of these additional equations described above is an arrest criterion on the proportional boundary conditions that controls the incremental crack advance.

- Stability criterion. The stability of the crack is an essential feature to be taken into account. Ideally (i.e., for a rigid testing machine), it is based on the change of the elastic energy with crack advance [53]. Such an assumption will be made in the following. In a real test, the rigidity of the testing machine may have to be taken into account to avoid unstable crack propagation [54]. Based on experience from real experiments [21], in our application case, the stabilising condition is set to be the rotational loading. The idea is to obtain the crack to open by edge rotation about the crack tip centre, which creates a relative compression zone in front of the crack thereby penalising its subsequent sudden propagation (Figure 5). This empirical procedure helps avoiding the crack to become unstable and to instantaneously propagate through the ligament. The corresponding loading is a rotation about a moving centre, which should be close to the crack tip. Represented as a superposition of tension and fixed centre rotation it could be written within a single linear constraint (Equation (2)). 

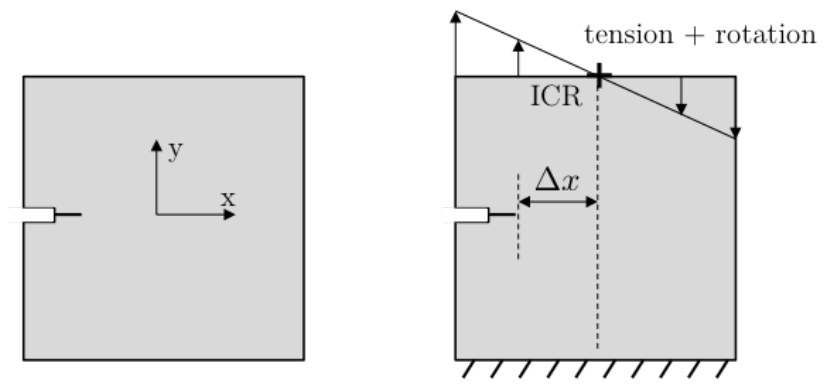

Figure 5: Example of a rotational loading stabilising crack propagation. The distance $\Delta x$ between the crack tip and the projected instantaneous centre of rotation (ICR) is introduced in the studied case

In the present case, the SIFs related to each boundary condition are computed with an LEFM model and the cracked geometry is updated using the X-FEM framework. The finite element model is enriched by adding degrees of freedom to the concerned elements, which take into account the displacement discontinuities through the crack faces and the stress field singularity in the vicinity of the crack tip. In this study, computations are performed by coupling $\mathrm{X}-\mathrm{FEM}$ with a level set propagation technique. One of the advantages of such an approach is that the computations are carried out only using the sound mesh.

In summary, the procedure consists of the following steps:

1. From the current and targeted crack path, compute the incremental crack direction, and hence the ratio $K_{I I} / K_{I}$.

2. Compute $[\boldsymbol{S}]$ matrix using a low-level model. Exploiting linearity and hence the superposition principle one computation of $\{\boldsymbol{k}\}$ is needed for each $b_{i}$.

3. The desired direction of propagation and the stability heuristic rule provide a direction for vector $\{\boldsymbol{k}\}$, from which a direction for the loading $\{\boldsymbol{b}\}$ is derived. (One may choose $K_{I}=K_{I c}$ to use a convention that can hold for the entire experiment, although it has no consequence.)

4. Until the crack has propagated by $d x$, apply the loading $\lambda(t)\{\boldsymbol{k}\}$ where $\lambda(t)$ is the magnitude of the loading along the direction $\{\boldsymbol{k}\}$, which is 
slowly increased with time.

5. Repeat steps 1-4 until the crack has propagated over the desired length.

\section{Proof of concept}

\subsection{Test-case}

To show the feasibility of the new method and the identification procedure, the test-case of a concrete sample with a sinusoidal crack path is considered.

315 This test-case is based on the multiple crack deflection experiments presented in Ref. 21. In these tests, concrete samples have been loaded in a hexapod using tension, shear and in-plane rotation as control variables. The cracks that initiated at the notch propagated slowly while the boundary conditions were manually changed in order to reorient the crack towards the desired angle. The 320 result of such test is a $Z$-shaped (i.e., zigzagged) cracked surface with multiple controlled turns. The following virtual experiment is proposed in the same spirit. Identical specimen geometry and type of loading will be used hereafter. The boundary conditions are applied on the upper face while the lower face is motionless. The kinematic loadings correspond to in-plane rigid body motions,

325 namely, global tension (i.e., translation along $y$ ), global shear (i.e., translation along $x$ ) and in-plane rotation.

The specimen is a $200 \times 200 \times 50 \mathrm{~mm}^{3}$ concrete parallelepiped with a $5 \times$ $25 \mathrm{~mm}^{2}$ notch (see Figure 6). 

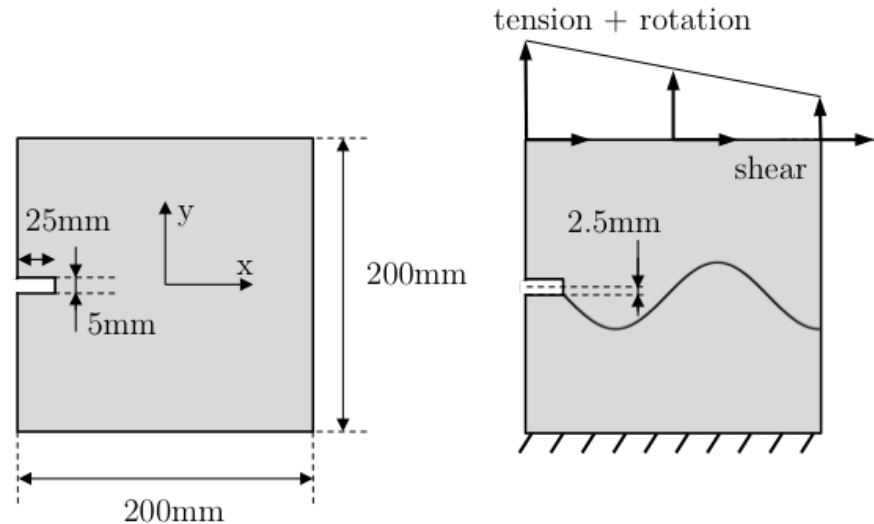

Figure 6: Test-case geometry and possible loading for the sinusoidal crack path

For this virtual test the chosen mechanical parameters are close to real concrete data 21]. The Young's modulus is set to $E=21 \mathrm{GPa}$, the Poisson's ratio to $\nu=0.2$, the tensile strength to $\alpha=3.9 \mathrm{MPa}$, the compressive strength to $\beta=8.5 \mathrm{MPa}$, the shear strength to $\gamma=3.25 \mathrm{MPa}$, and the Griffith energy to $G_{f}=100 \mathrm{~J} / \mathrm{m}^{2}$. The input crack path $y_{\text {input }}(x)$ is a sinusoidal function with 1.25 period and an amplitude of $15 \mathrm{~mm}$ with an offset of $-2.5 \mathrm{~mm}$ in order to start in the corner of the notch

$$
y_{\text {input }}(x)=-15 \sin \left[2 \pi \frac{1.25}{175}(x+75)\right]-2.5
$$

The virtual test follows the same principle (see Figure 7) as the real one, namely, the numerical control is the same as used in real tests, the experimental loading device is replaced by a numerical simulation performed using a damage model in order to obtain realistic responses. The assessment of the crack tip is also performed by numerical means. For each iteration $i$, the crack propagates because of the $i$-th loading and the final $i$-th position of the tip is the input in the control part for iteration $i+1$. Finally the sample is unloaded and the boundary conditions are computed for the next iteration. 


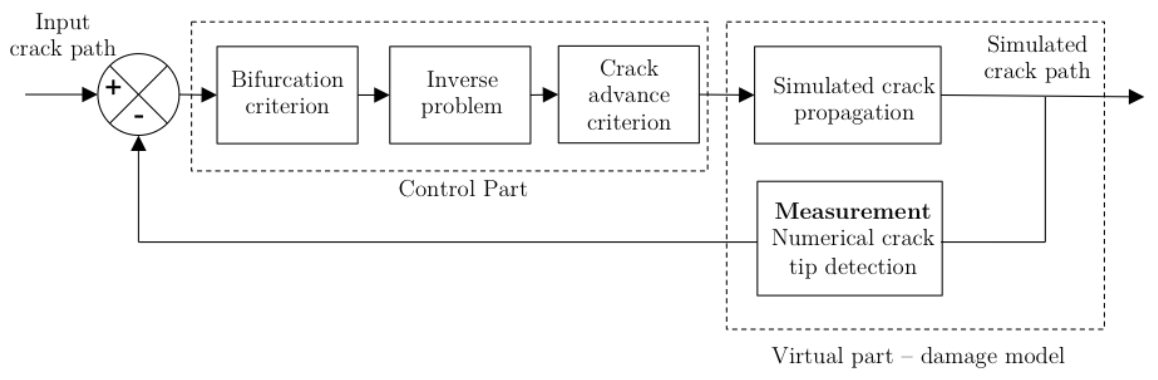

Figure 7: Block diagram of virtual 3rd generation tests

Because crack propagation is simulated with a damage model, the crack tip position is numerically found by analysing damage field contours. For a virtual test, the testing machine, the specimen and the measurement can be seen as a black box where boundary conditions are sent in and a crack path is obtained.

\subsection{Deflection criterion}

In the following, the simulated crack path and its position will be considered as a true representation of crack tip propagation and the numerical results as experimental reference. The chosen deflection criterion is the maximum normal stress. The crack tip is assumed to turn in the direction where the normal stress ( $\sigma_{\theta \theta}$ in a local crack frame) is maximum. The local SIF ratio that has to be applied to the specimen is thus given by (see Equation (3)

$$
\frac{K_{I I}}{K_{I}}=\frac{\sin (\theta)}{1-3 \cos (\theta)}
$$

\subsection{Inverse problem}

The linear system that uses the boundary conditions to deflect, propagate and stabilise the crack is presented. The three needed boundary conditions are on the upper face tension $b_{1}$, shear $b_{2}$ and a plane rotation about the upper face centre $b_{3}$. In this test-case, the stabilization of the crack path is controlled with a moving Instantaneous Centre of Rotation (ICR) at a determined $x$ position. The $x$ position of the centroid, which depends on tension and rotation, enables 
tensile and compressive zones to be created in the sample. In order to stabilise crack propagation, the ICR has been located at a horizontal distance $\Delta x$ from the crack tip. This distance has to be chosen with caution. It is bounded by

- the prediction of models (i.e., with LEFM, $\Delta x \in[0, \infty]$ to ensure $K_{I}>0$ at the current crack tip)

- the real experimental behaviour (e.g., intermittent propagation of the crack, heterogeneity)

- the capacity of the machine (i.e., the maximum level of tension, compression and torque the hexapod is able to perform)

- the material behaviour in other parts of the sample (i.e., damage on the compressive zone and on the edges)

From previous experience [21], it has been chosen to set this horizontal distance from the crack tip $\Delta x=58 \mathrm{~mm}$. The position $\ell(i)$ of the ICR is thus updated for each crack propagation by $\ell(i)=C(i)+\Delta x$ where $C(i)$ is the horizontal crack position for the corresponding time step, and $\ell(0)=-16.5 \mathrm{~mm}$. This condition is rewritten as

$$
b_{1}+\ell(i) b_{3}=0
$$

The linear system (7) that has to be inverted to find the value of the boundary conditions reads

$$
\left\{\begin{array}{c}
K_{I} \\
K_{I I} \\
0
\end{array}\right\}=\left[\begin{array}{ccc}
S_{I}^{1} & S_{I}^{2} & S_{I}^{3} \\
S_{I I}^{1} & S_{I I}^{2} & S_{I I}^{3} \\
1 & 0 & \ell(i)
\end{array}\right] \quad\left\{\begin{array}{c}
b_{1} \\
b_{2} \\
b_{3}
\end{array}\right\}
$$


When inverting and solving the system with Equation (7), the sought ratio of boundary conditions is obtained

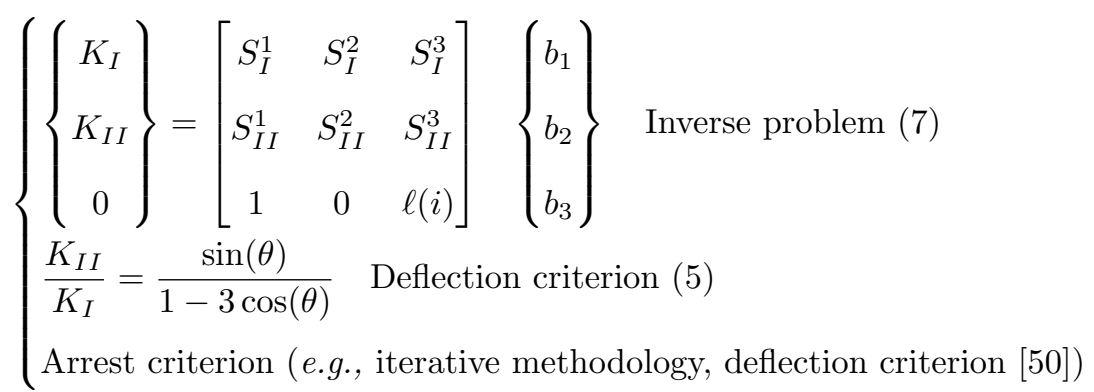

In the following test-case, the advance of the crack has been set according to the arrest criterion. This corresponds to an advance of the crack by $d x=6.6 \mathrm{~mm}$ per step $C(n)=C(0)+n \times d x$. A graphical representation of the system is shown in Figure 8 in the $\left(K_{I}, K_{I I}\right)$ plane when normalised by the toughness $K_{I c}$. The influence of the two boundary conditions is different and complementary, and a linear combination has to be chosen to get the adequate SIF ratio.

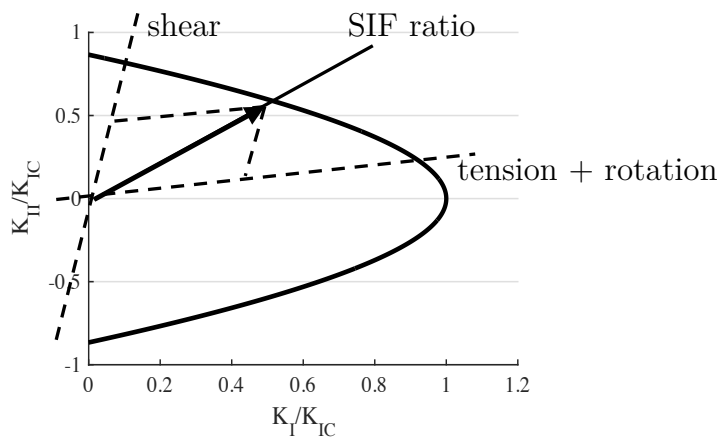

Figure 8: Fracture threshold (maximum normal stress criterion [50]) and influence of the boundary conditions

\subsection{Mechanical loading and measurement}

For the considered virtual test, a damage model simulates crack propagation. The computation is performed with a non-local damage model [8, 55] suitable for quasi brittle materials like concrete. The model considers the growth of 
damage as an irreversible process, differentiates compressive/tensile responses, and does not take into account crack closure in the current formulation.

Damage is described by a scalar field $a(\mathbf{x})$ defined on the entire sample, $a=0$ corresponding to sound material and $a=1$ to a fully damaged state. With the increase in damage the stiffness of the material, $A(a)$, decreases (i.e., $A(0)=1$ and $A(1)=0)$. The stress/strain relationship takes into account stiffness loss

$$
\boldsymbol{\sigma}=A(a) \boldsymbol{E}: \boldsymbol{\epsilon}
$$

where $\boldsymbol{E}$ is the stiffness tensor. The following potential functional is used to describe the material state

$$
\Phi(\boldsymbol{\epsilon}, a)=A(a) \Gamma(\boldsymbol{\epsilon})+k a+\frac{c}{2}(\nabla a)^{2}
$$

where $\boldsymbol{\epsilon}$ is the infinitesimal strain tensor, $k a$ the dissipated energy, and $c$ the parameter that controls the size of the damage localization zone $D$. The $1 \mathrm{D}$ analytical solution [55] opens the way for the parameters $k$ and $c$ to be identified, which can be linked to Griffith energy $G_{f}$ by

$$
k=\frac{3}{4} \frac{G_{f}}{D} \quad c=\frac{3}{8} D G_{f}
$$

The $\Gamma$ function is expressed as

$$
\Gamma(\boldsymbol{\epsilon})=\left(c_{T} \operatorname{tr}(\boldsymbol{\epsilon})+\sqrt{c_{H} t^{2}(\boldsymbol{\epsilon})+c_{S} \frac{3}{2} \boldsymbol{\epsilon}^{\text {dev }}: \boldsymbol{\epsilon}^{\boldsymbol{d e v}}}\right)^{2}
$$

where $c_{H}, c_{T}$ and $c_{s}$ are numerical parameters that can be identified from three physical measurements, namely, the triplet of tensile strength, compressive strength and shear strength.

The yield surface is obtained by deriving the functional $\Phi$ with respect to the damage field $a(\mathbf{x})$

$$
f(\boldsymbol{\epsilon}, a)=-\frac{\partial \Phi}{\partial a}
$$

The consistency (Kuhn-Tucker) conditions read

$$
f \leq 0, \dot{a} \geq 0, \dot{a} f=0
$$


The elastic domain $f(\boldsymbol{\epsilon}, 0)=0$ is an ellipsoid of revolution defined by $\Gamma(\boldsymbol{\epsilon})=$ constant. The real yield surface [56] of concrete is described in the tension-shear domain by adjusting the model parameters (Figure 9

$$
\begin{aligned}
c_{T} & =\sqrt{\frac{E}{2}} \frac{\alpha}{1-2 \nu}\left(\frac{1}{2 \alpha}-\frac{1}{2 \beta}\right) \\
c_{H} & =\frac{\alpha^{2} E}{(1-2 \nu)^{2}}\left[\left(\frac{1}{2 \alpha}-\frac{1}{2 \beta}\right)^{2}-\frac{1}{3 \gamma^{2}}\right] \\
c_{S} & =\frac{E \alpha^{2}}{6 \gamma^{2}(1+\nu)^{2}}
\end{aligned}
$$

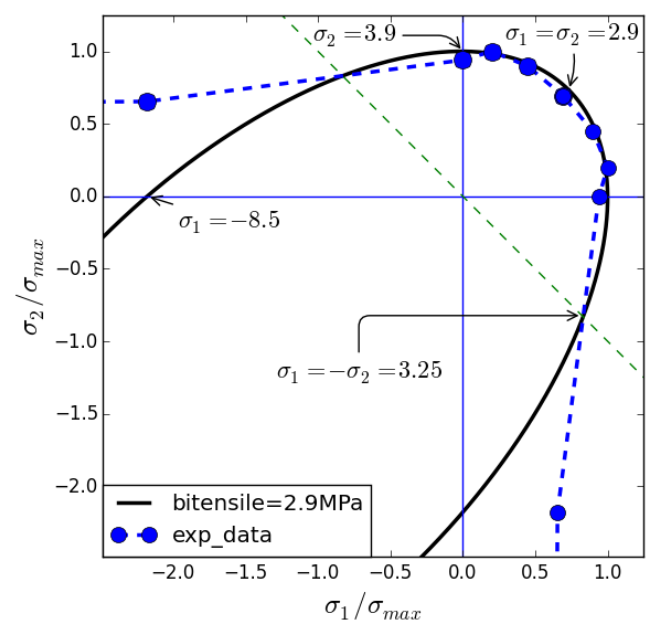

Figure 9: Elastic domain (plane stress) shape in the bitensile quadrant of the experimental data for concrete 56 .

Last, the stiffness loss function $A(a)$ defines the post-peak behaviour of the material. It is written by using two parameters

$$
A(a)=\frac{(1-a)^{2}}{(1-a)^{2}+m a(1+p a)}
$$

where $m$ defines the initial damage rate

$$
m=\frac{3}{2} \frac{E G_{f}}{D \alpha^{2}}
$$


The parameter $p$ is more important as it influences the size of the process zone. The identification of the latter is very challenging for classical fracture experiments. That is why the identification presented in the next section will deal with this scalar parameter.

For the computations, the numerical parameter $D$ has to be adapted to the size of the mesh to cover at least 3 elements inside the damaged zone of size $2 \times D$ (i.e., finer meshes are not needed as the results converge quickly to a cohesive zone response [55]). In the current study $D$ is set to $1 \mathrm{~mm}$ and the length scale $p$ to 1.5 . The mesh is composed of 31,296 6-noded triangular (T6) elements with an average size equal to $1 \mathrm{~mm}$.

The damage model is implemented in Code_Aster [57]. The measurement of the crack tip is obtained at each step from the damage field with the Code_Aster module Post_Endo_Fiss [58. With this method, the crack path is detected when the damage level is greater than a characteristic threshold. During each iteration, the new crack tip is detected and injected in the control part. Consequently the crack geometry has to be updated after each propagation increment. The threshold on the damage variable to detect the crack is set to $a=0.1$.

In this study with a numerical simulation of the crack path, the arrest criterion is an iterative method based on a certain amount of damage increase per increment. This is possible to obtain since a numerical simulation is used and coupled with the damage control continuation method implemented in Code_Aster [59]. This functionality is very convenient since the position of 400 the crack tip cannot be known on the fly during loading with the FE code. The crack tip position and the damage level are strongly linked with the crack propagation length per iteration, as will be shown in the next section.

\subsection{Results}

Once the 33 reorientation iteration steps have converged, the last thin ligament tends to break in an unstable manner and can no longer be controlled. The result obtained after all 33 steps is shown for the horizontal and vertical displacement fields in Figure 10 and the crack propagation path in Figure 11 . 


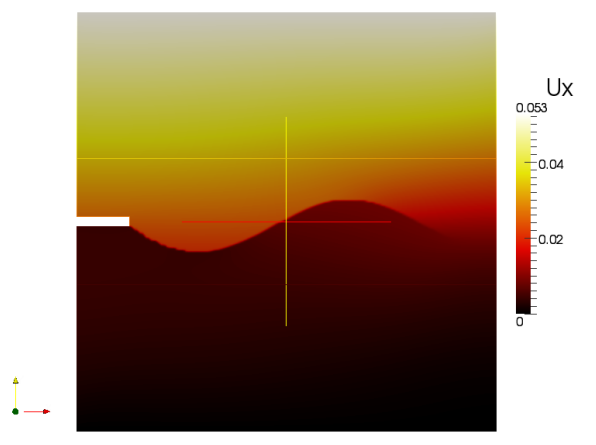

(a)

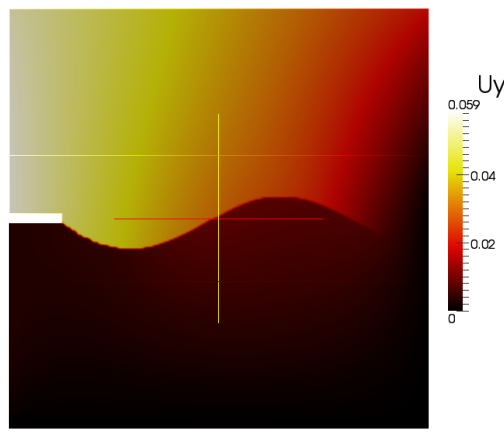

(b)

Figure 10: Displacement field at the end of the virtual sine test. (a) Horizontal ( $x$-axis), and (b) vertical ( $y$-axis) components

In Figure 11, the target crack path and that obtained by the simulated procedure (red circle) are compared. Even if there is a visible deviation between both crack paths, it is observed that the simulated path is close to the target. The mean relative error is $6.3 \%$, which is deemed acceptable.

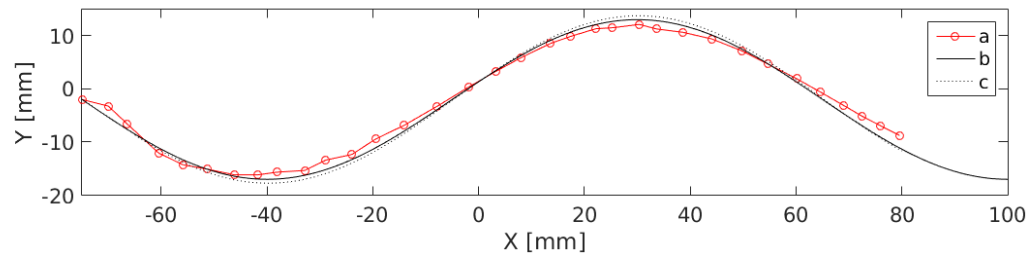

Figure 11: Comparison between (a) the measured crack path, (b) the target crack path, and (c) the input crack (never reached because of the step discretization)

The maximum displacement of the top left and right corners of the sample applied for each increment before unloading is reported in Figure 12. Even if a global sinusoidal shape can be seen on the shear boundary conditions, the precise loading history is not trivial and hence not easily guessed a priori. 


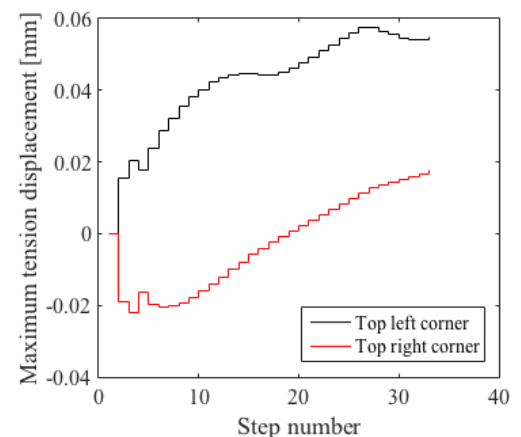

(a)

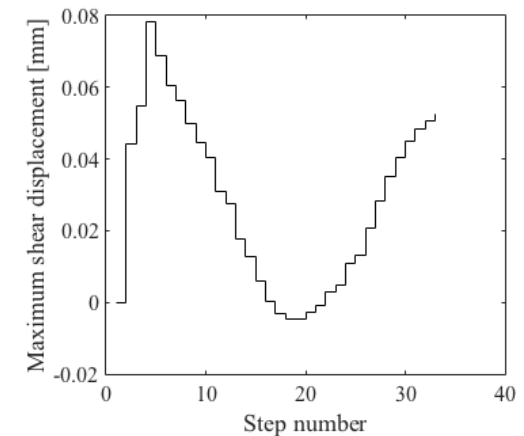

(b)

Figure 12: Maximum applied displacement at the top left and right corners of the sample vs. step number. (a) Tension on the top left corner (positive values) and on the top right corner, and (b) shear (uniform)

The crack length for each iteration is plotted in Figure 13. The increase of the crack length and the variation of boundary conditions mean that the crack continuously turns during the experiment.

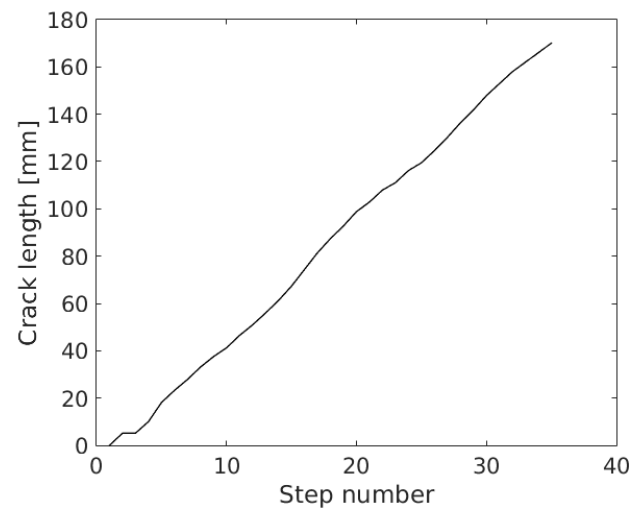

Figure 13: Total crack length vs. step number

The length of the crack vs. the number of increments is close to a linear curve. The mean crack advance is found to be $\approx 5 \mathrm{~mm}$ per iteration. This length does not exactly correspond to the advance of the $\mathrm{ICR}(\approx 6.6 \mathrm{~mm})$. Even if the tensile 
gradient slightly decreases during the test, it is still important to stabilise the crack. During propagation, the mean absolute value of the deflection angle is $\langle|\theta|\rangle=6^{\circ}$, i.e., the crack does not rotate much for each iteration because of small propagation increments and the rather smooth prescribed crack path.

With the help of the iterative procedure the inverse damage problem is solved recursively, i.e., the boundary conditions (Figure 12) could be directly applied to the sample from the start until the end (i.e., 33rd step) to obtain the desired sinusoidal path if the behaviour of the machine and the specimen were exactly those of the numerical simulation.

The question of the relationship between the 'low-level' and 'high-level' models is worth mentioning. In the present case, it is natural to observe that if the internal length scale of the model is much smaller than all characteristic sizes of the problem (here the smallest crack path radius of curvature), the highlevel model becomes identical to the low-level one, that is LEFM. Hence a very good control is expected, unless precisely when the internal length scale plays a significant role, and hence the present approach naturally provides a very discriminating tool to reveal such model enrichment as explored in the following subsection.

However, one may wonder whether a cruder 'low-level' model may still provide a viable route to experimentally control such a sinuous crack path. To this end, a very poor version of the 'low level' model has been used to test the control principle. Instead of an LEFM prediction, a simple proportional-integral (PI) controller with incremental change of the shear stress and a constantly growing tension/rotation permits us to drive the crack as desired during the first 12 steps. However the inaccuracies of this low level model produce spurious damage patterns from which a secondary crack may branch whenever the main crack path reaches some unfavourable direction. More details are shown in Appendix A. The feasibility of the iterative control of a crack propagation, even though 450 limited, is proved. 


\section{Application to identification}

The aim of the testing procedure is to be sensitive enough to the model parameters to enable for their identification. A rough estimate of the sensitivity to the length scale can be directly obtained from the analysis of the crack path trajectory with respect to the studied parameter variation. As shown in Figure 14 the crack path is sensitive to the length scale parameter $p$, but it is difficult to assess its best fit value, as its variation is really important (i.e., a factor of 3$)$.

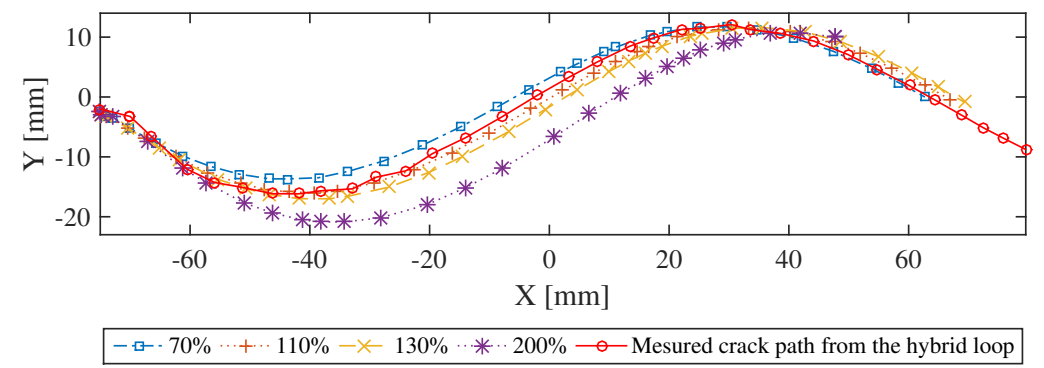

Figure 14: Crack path sensitivity to the length scale parameter $p$

For a more quantitative analysis, an identification procedure must be performed. The previous test-case will be used for identification purposes with artificial noise added in order to prove its robustness.

\subsection{Global DIC}

In practice, a global DIC approach [60] will be used to measure the displacement field for identification purposes [61. It consists of the registration of an image $f(\boldsymbol{x})$ in the reference configuration and a series of pictures $g(\boldsymbol{x}, t)$ in the deformed configuration indexed by time $t$. The correlation code minimises 
the sum of squared differences between the deformed image corrected by the measured displacement $\boldsymbol{u}(\boldsymbol{x}, t)$ and the reference image

$$
\chi_{f}^{2}(t)=\frac{1}{2 \gamma_{f}^{2}|\Omega|} \sum_{\Omega}\left((g(\boldsymbol{x}+\boldsymbol{u}(\boldsymbol{x}, t), t)-f(\boldsymbol{x}))^{2}\right.
$$

with respect to the nodal displacement vector $\left\{\boldsymbol{u}_{m}\right\}$ of a finite element mesh defined by the shape functions $\Phi_{i}$ and unknown degree of freedom $u_{i}$ such that $\boldsymbol{u}(\boldsymbol{x}, t)=\sum_{i} \boldsymbol{\Phi}_{i}(\boldsymbol{x}) u_{i}(t)$, where $\boldsymbol{x} \in \Omega$ is any considered pixel, $\Omega$ the Region Of Interest (ROI) and $\gamma_{f}$ the standard deviation (expressed in grey levels) of the Gaussian white noise assumed to affect each image independently (including the reference one, which is responsible for the factor 2).

The minimization of this functional is solved by successive linearisations and corrections using modified Gauss-Newton scheme 62

$$
[\boldsymbol{M}]\left\{\delta \boldsymbol{u}_{m}\right\}=\{\boldsymbol{b}\}
$$

where $[\boldsymbol{M}]$ is the DIC matrix, $\left\{\delta \boldsymbol{u}_{m}\right\}$ the vector gathering all corrections to the measured degrees of freedom, and $\{\boldsymbol{b}\}$ the residual column vector, which cancels out when the grey level conservation is satisfied everywhere. Since the measured displacement field is corrupted by noise, the DIC matrix is related to the covariance matrix $\left[\boldsymbol{C}_{\boldsymbol{u}}\right]$ of the measured degrees of freedom by [60]

$$
\left[\boldsymbol{C}_{\boldsymbol{u}}\right]=2 \gamma_{f}^{2}[\boldsymbol{M}]^{-1}
$$

Once a discretization has been chosen for displacement field measurements, and the level of acquisition noise is known, this derivation gives access to the sensitivity of the measured degrees of freedom to acquisition noise 62.

\subsection{Weighted FEMU}

Several identification methods have been adapted or developed to quantitatively use full field measurement results to calibrate material parameter [63, 61]. The most used method is Finite Element Model Updating (FEMU), which is based on the minimization of the differences between the measured quantities (i.e., forces $\boldsymbol{F}_{\boldsymbol{m}}$ and displacement fields $\boldsymbol{u}_{\boldsymbol{m}}$ ) and the corresponding computed 
quantities from a numerical simulation, namely, $\boldsymbol{F}_{\boldsymbol{c}}$ and $\boldsymbol{u}_{\boldsymbol{c}}$. This simulation, which utilises a constitutive model, is performed with Dirichlet boundary conditions extracted from, say, DIC analyses.

Weighted displacement-based FEMU (i.e., FEMU-U) consists in computing the set of (dimensionless) constitutive parameters $\{\boldsymbol{p}\}$ that minimises the functional $\chi_{u}^{2}[62$

$$
\chi_{U}^{2}=\frac{1}{N_{u}}\left\{\boldsymbol{u}_{\boldsymbol{m}}-\boldsymbol{u}_{\boldsymbol{c}}\right\}\left[\boldsymbol{C}_{\boldsymbol{u}}\right]^{-1}\left\{\boldsymbol{u}_{\boldsymbol{m}}-\boldsymbol{u}_{\boldsymbol{c}}\right\}
$$

where $\chi_{U}^{2}$ is the weighted squared difference between the measured and calculated displacements, and $N_{u}$ the total number of kinematic degrees of freedom. The weighted chi-squared error therefore refers to the $[\boldsymbol{M}]$-norm associated with the DIC matrix (up to a constant prefactor $1 /\left(2 \gamma_{f}^{2}\right)$ ).

The reaction forces may also be measured and computed. Thus, the same approach is followed with the reaction forces for which $\chi_{F}^{2}$ is minimised (FEMUF) 62

$$
\chi_{F}^{2}=\frac{1}{N_{F}}\left\{\boldsymbol{F}_{\boldsymbol{m}}-\boldsymbol{F}_{\boldsymbol{c}}\right\}\left[\boldsymbol{C}_{\boldsymbol{F}}\right]^{-1}\left\{\boldsymbol{F}_{\boldsymbol{m}}-\boldsymbol{F}_{\boldsymbol{c}}\right\}
$$

485 the measured load (in the present case it is assumed that the load measurements are uncorrelated so that $\left[\boldsymbol{C}_{\boldsymbol{F}}\right]=\gamma_{F}^{2}[\boldsymbol{I}]$, with $\gamma_{F}$ the standard load uncertainty and $[\boldsymbol{I}]$ the identity matrix).

The identification based on the combined displacement field and reaction force (FEMU-UF) [64 is achieved by minimising the global functional $\chi_{U F}^{2}$ in a Bayesian framework 62 .

$$
\chi_{U F}^{2}=\frac{N_{u}}{N_{u}+N_{F}} \chi_{u}^{2}+\frac{N_{F}}{N_{u}+N_{F}} \chi_{F}^{2}
$$

\subsection{Comparison of two different tests}

The so-called Nooru-Mohammed tests [65] have become a reference for mixed mode propagation of cracks in quasi brittle samples. One of these crack paths will be chosen to be compared with the sinusoidal path obtained with the present method. The input data for identification purposes are the measured boundary 
conditions of the sample (i.e., force and displacement field). These boundary conditions applied to the top and bottom surface are known for a NooruMohammed test. The material and behaviour are the same as in the sinusoidal test-case. A double notched sample is analysed. The most used crack path of all Nooru-Mohammed tests is the number 5. As in the real Nooru-Mohammed test, the load starts with shear up to $10 \mathrm{kN}$, then the sample is loaded with a tensile displacement while maintaining constant the shear force. This load leads to a curved crack path in mixed mode I and II.

\begin{tabular}{|c|c|c|c|c|c|c|c|}
\hline Parameter & $p$ & $\nu$ & $\beta$ & $G_{f}$ & $E$ & $\gamma$ & $\alpha$ \\
\hline Units & - & - & $(\mathrm{MPa})$ & $\left(\mathrm{J} / \mathrm{m}^{2}\right)$ & $(\mathrm{GPa})$ & $(\mathrm{MPa})$ & $(\mathrm{MPa})$ \\
\hline Value & $1.05-3$ & 0.2 & 8.5 & 100 & 20 & 3.25 & 3.9 \\
\hline Number & 1 & 2 & 3 & 4 & 5 & 6 & 7 \\
\hline
\end{tabular}

The predicted behaviour is simulated with the non-local damage law presented previously. In the following the most difficult parameter to identify, namely, the length scale $p$ that controls the size of the process zone, is studied. The other parameters (i.e., $E, \nu, \alpha, \beta, \gamma$ and $G_{f}$ ), which can be more easily identified with standard tests are assumed to be known. The values of the parameters are reported in Table 1. Even though the experiment has not been performed yet, it is possible to analyse its sensitivity to various quantities (e.g., material parameters, sample geometry, loading path). The sensitivity analysis can be performed on any of the three identification residuals (i.e., $\chi_{u}^{2}$, $\chi_{F}^{2}, \chi_{u F}^{2}$, see Refs. [66, 67]). In the present case, the dimensionless Hessian is computed when only kinematic data are considered

$$
\left[\boldsymbol{H}_{\boldsymbol{u}}\right]=\left[\boldsymbol{S}_{\boldsymbol{u}}\right]^{t}\left[\boldsymbol{C}_{\boldsymbol{u}}\right]^{-1}\left[\boldsymbol{S}_{\boldsymbol{u}}\right]
$$

with

$$
\left\{\boldsymbol{S}_{\boldsymbol{u}}\right\}_{i}=\left\{\boldsymbol{u}_{\boldsymbol{c}}\left(p_{i}+\delta p\right)\right\}-\left\{\boldsymbol{u}_{\boldsymbol{c}}\left(p_{i}\right)\right\}
$$

where $\left\{\boldsymbol{S}_{\boldsymbol{u}}\right\}_{i}$ denotes the column vector of displacement sensitivities with respect to the $i$-th material parameter when $\delta p$ is equal to $10 \%$ of $p_{i}$. The eigenvalues 
of $\left[\boldsymbol{H}_{\boldsymbol{u}}\right]$ are very helpful to probe the sensitivity of a given experimental configuration to the chosen parameters $\{\boldsymbol{p}\}$ [66]. If only the length of the process zone parameter (i.e., $p$ ) is studied, the diagonal component $p$ of the dimensionless Hessian $\left[\boldsymbol{H}_{\boldsymbol{u}}\right]$ is to be compared. In the present case, its level is equal to $1.5 \times 10^{-3}$ for the Nooru-Mohammed test, and $9.0 \times 10^{-2}$ for the proposed experiment. The norm of the sensitivity for the length scale parameter is 59 times higher for the sinusoidal test, which proves the interest of such test.

More globally, the spectrum of eigenvalues associated with the dimensionless Hessian $\left[\boldsymbol{H}_{\boldsymbol{u}}\right]$ can be compared to probe the global sensitivity of the two experiments to the whole set of material parameters for the chosen damage model (i.e., the higher any eigenvalue, the more sensitive the experiment). Figure 15 shows the spectrum for the two configurations. On average there is a two order of magnitude difference in favour of the new configuration. These results show that the new configuration is significantly more sensitive to the material parameters.

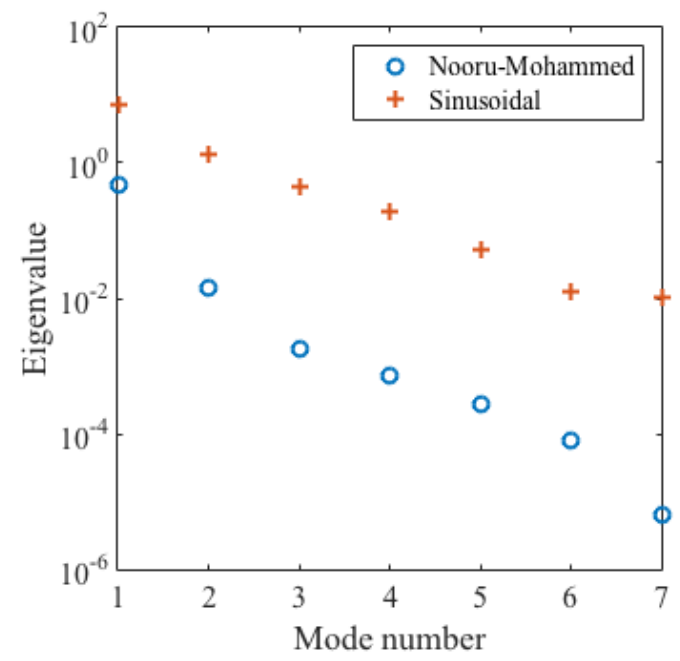

Figure 15: Comparison of the 7 eigenvalues of $\left[\boldsymbol{H}_{\boldsymbol{u}}\right]$ with a sinusoidal test and NooruMohammed test

The coupling between the parameters is analysed with the covariance nor- 
malised by the diagonal terms. The corresponding correlation matrix is formed (with no index summation)

$$
\left[\widetilde{H}_{u}^{-1}\right]_{i j}=\frac{\left[H_{u}^{-1}\right]_{i j}}{\sqrt{\left[H_{u}^{-1}\right]_{i i}} \sqrt{\left[H_{u}^{-1}\right]_{j j}}}
$$

For the sinusoidal crack path the correlation matrix shows an anti-correlation

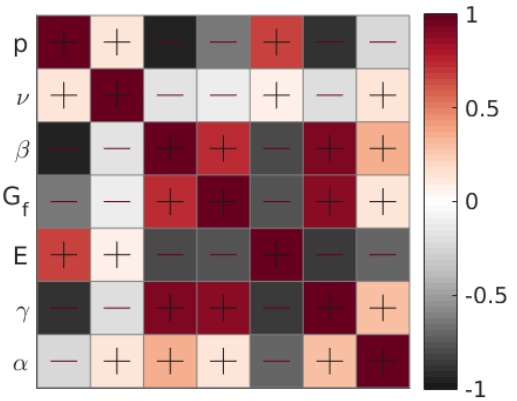

(a)

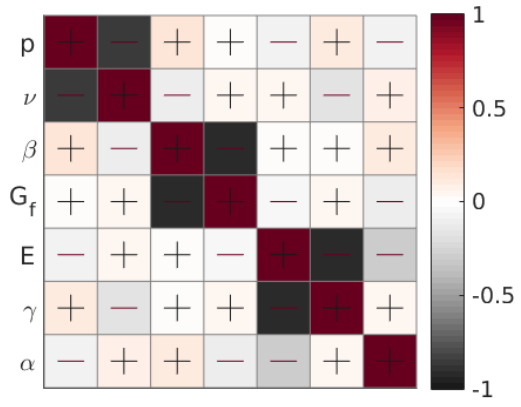

(b)

Figure 16: Correlation matrix for (a) the Nooru-Mohammed test (b) the sinusoidal experiment

To probe the noise sensitivity of the proposed identification procedure with the sinusoidal test-case, displacement uncertainties $\eta_{u}$ and force uncertainties $\eta_{F}$ have been added to the numerical noiseless results prior to the identification step. For the displacement field, a DIC computation of a reference image representative of the proposed test (extracted from a real test [21]) is run with the same image for which white Gaussian grey level noise is added. The grey level noise has a standard deviation $\gamma_{f}$ equal to $0.6 \%$ of the dynamic range (i.e., difference between maximum and minimum grey levels) of the reference image. The corresponding nodal displacement due to acquisition noise $\left\{\boldsymbol{\eta}_{\boldsymbol{u}}\right\}$ is added to the 'measured' displacement field $\left\{\boldsymbol{u}_{\boldsymbol{c}}\right\}$. To summarise, the displace- 
ment error is obtained with the normalised quadratic difference between the reference displacement field considered as the measured one (with parameter $p$ and corrupted by the noise) and the computed field (with $p+\delta p$ ).

For the force uncertainty, $\gamma_{F}$ is assumed to be proportional to the magnitude of the load level with a coefficient $\rho_{1}^{2}[68$. A minimum uncertainty for the load cells is included whose variance $\rho_{0}^{2}$ is independent of the load level so that the load uncertainty reads

$$
\gamma_{F}^{2}=\rho_{1}^{2}|F|^{2}+\rho_{0}^{2}
$$

where the parameters, which are representative of nowadays cameras and load cells, are given in Table 2 .

Table 2: Parameters for the analysis of noise sensitivity

\begin{tabular}{|c|c|c|}
\hline$\gamma_{f}$ & $\rho_{1}$ & $\rho_{0}$ \\
\hline $0.6 \%$ of 8-bit images =2 grey levels & $4 \times 10^{-4}$ & $30 \mathrm{~N}$ \\
\hline
\end{tabular}

For different values of the parameter $p$ ranging from $70 \%$ to $200 \%$ of the initial reference value (i.e., $p=1.5$ ), the FEMU-UF functional is plotted in Figure 17. The shape of the functional for the sinusoidal path is much steeper than for Nooru-Mohammed's case. This means that the sinusoidal crack path is more sensitive to the length scale $p$ and less to noise. Hence the identification procedure will converge more precisely and faster [69].

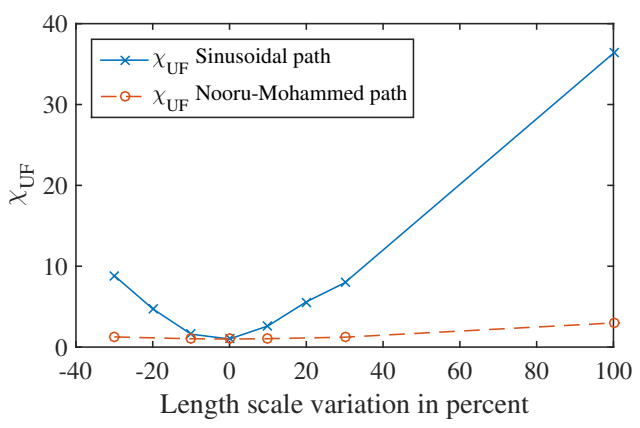

Figure 17: Weighted norm of the error $\chi_{U F}$ as a function of the length of the process zone for the two test configurations 


\section{Conclusion}

545 quantities of interest (e.g., SIF, crack tip position) in order to validate models and calibrate their parameters. A virtual test-case has shown the feasibility of the described method by following a sinusoidal crack path on a concrete sample. The loading was in tension, shear and in-plane rotation and crack propagation was simulated with a non-local damage model. The extracted boundary conditions enable the sensitivity of this crack path to be assessed relative to a more standard crack path for mixed mode crack propagation in quasi brittle materials. It has been shown that the crack path obtained with the proposed method was about two orders of magnitude more sensitive especially for the length scale identification.

The sinusoidal shape has been arbitrarily selected and was not optimised for identification purposes. The use of the proposed virtual method allows different crack paths to be analysed very quickly. An optimization step of the crack path for the identification of a specific parameter can be performed based on the presented framework [66]. Last, achieving real 3rd generation tests is the next step of the present study to calibrate internal length scales of damage models, which are very difficult to estimate with current tests and procedures.

\section{Acknowledgement}

The support of CNRS (French National Centre for Scientific Research) for the CONCRET project is gratefully acknowledged.

\section{Appendix A}

To go along the same lines as Section 3 but exploring yet a cruder example of low level prediction, a poor version of the control model has been tested in the same spirit as the manual tests performed by Carpiuc et al. [21]. Instead of working with an LEFM prediction, the control was performed by an incremental 
change of the shear component $U_{s}$ and a constantly increasing tensile/rotation component (Figure 18).

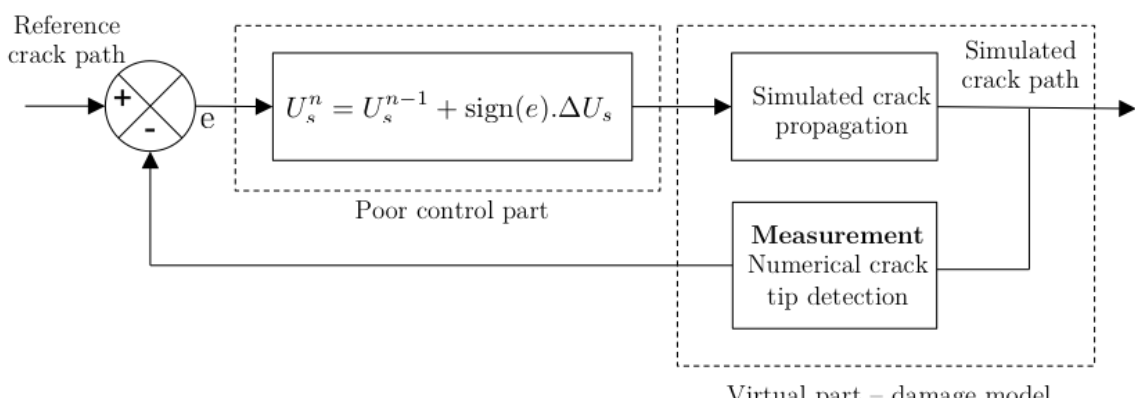

Figure 18: Control loop of the crack path with a low level (PI) control model. $U_{s}$ is the shear displacement boundary condition, which is mainly responsible for the deflection of the crack, and $\Delta U_{s}$ an elementary variation of the shear component

The results are that the deflection of the crack is less smooth and creates bifurcation zones. However, for small increments, the control of the 'high level model' was performed by a simple proportional integral (PI) corrector (as a qualitative illustration, see Figure 19p. Such a control strategy provides an acceptable response for the main crack up to an inflection point of the trajectory. 


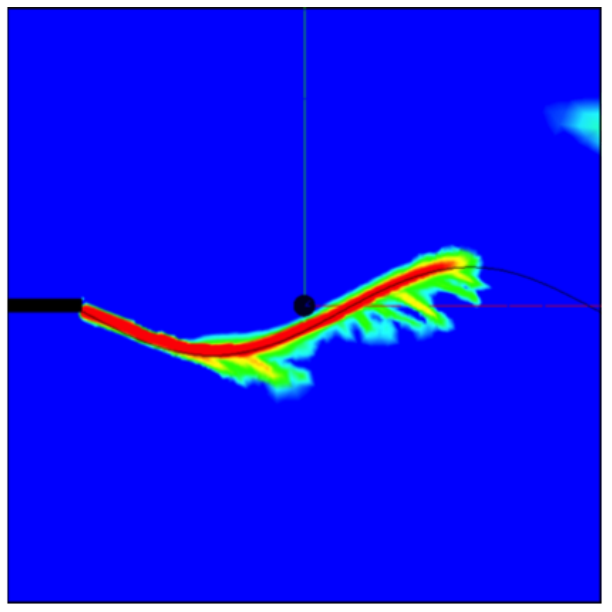

Figure 19: Damage field (red is for the damage variable greater than 0.95) with multiple branching zones for a crack controlled with the 'low level' (PI control) model

However, because of the distance to the actual physics of the problem, more damage accumulates on the concave side of the crack. Past the inflection point for the main crack trajectory, these damaged zones act as secondary branching points, and thus prevent us from controlling the crack path until its very end. This result illustrates the benefit of having a low level model that is close enough to the expected ground truth.

Last, let us note that for the inversion of a non-linear control model, the 585 implementation of a Gauss-Newton routine, for example, may solve the problem with successive updated sensitivities on the boundary conditions. 


\section{References}

[1] Y. Rashid, Ultimate strength analysis of prestressed concrete pressure vessels, Nuclear Engineering and Design 7 (4) (1968) 334-344.

[2] R. de Borst, P. Nauta, Nonorthogonal cracks in a smeared finite element model, Engineering Computations 2 (1) (1985) 35-46.

[3] J. Mazars, A description of microscale and macroscale damage of concrete structures, Engineering Fracture Mechanics 25 (5-6) (1986) 729-737.

[4] G. Pijaudier-Cabot, Z. Bazant, Nonlocal Damage Theory, ASCE Journal of Engineering Mechanics 113 (10) (1987) 1512-1533.

[5] R. Peerlings, R. de Borst, W. Brekelmans, J. de Vree, Gradient-enhanced damage for quasi-brittle materials, International Journal for Numerical Methods in Engineering 39 (1996) 3391-3403.

[6] R. Desmorat, F. Gatuingt, F. Ragueneau, Nonlocal anisotropic damage model and related computational aspects for quasi-brittle materials, Engineering Fracture Mechanics 74 (10) (2007) 1539-1560.

[7] M. Jirásek, Damage and Smeared Crack Models, Springer Vienna, Vienna, 2011, pp. 1-49.

[8] E. Lorentz, V. Godard, Gradient damage models: Toward full-scale computations, Computer Methods in Applied Mechanics and Engineering 200 (2122) (2011) 1927-1944.

[9] D. Xenos, D. Grégoire, S. Morel, P. Grassl, Calibration of nonlocal models for tensile fracture in quasi-brittle heterogeneous materials, Journal of the Mechanics and Physics of Solids 82 (2015) 48-60.

[10] A. Hillerborg, M. Modeer, P. Petersson, Analysis of crack formation and crack growth in concrete by means of fracture mechanics and finite elements, Cement Concrete Research 6 (1976) 773-782. 
[11] A. Pandolfi, S. Conti, M. Ortiz, A recursive-faulting model of distributed damage in confined brittle materials, Journal of the Mechanics and Physics of Solids 54 (9) (2006) 1972-2003.

[12] P. Carrara, L. D. Lorenzis, A coupled damage-plasticity model for the cyclic behavior of shear-loaded interfaces, Journal of the Mechanics and Physics of Solids 85 (2015) 33-53.

[13] Z. Bažant, B. Oh, Crack band theory for fracture of concrete, Matériaux et Construction 16 (3) (1983) 155-177.

[14] G. Francfort, J. Marigo, Rivisiting brittle fracture as an energy minimization problem, Journal of the Mechanics and Physics of Solids 46 (1998) $1319-1342$.

[15] A. Delaplace, G. Pijaudier-Cabot, S. Roux, Progressive damage in discrete models and consequences on continuum modelling, Journal of the Mechanics and Physics of Solids 44 (1) (1996) 99-136.

[16] F. Hamon, Modèle d'endommagement de Mazars, EDF R \& D (http://www.code-aster.org/doc/v11/fr/man_r/r7/r7.01.08.pdf), 2013.

[17] C. Le Bellégo, J.-F. Dubé, G. Pijaudier-Cabot, B. Gérard, Calibration of nonlocal damage model from size effect tests, European Journal of Mechanics - A/Solids 22 (1) (2003) 33-46.

[18] C. Iacono, L. Sluys, J. van Mier, Calibration of a higher-order continuum model using global and local data, Engineering Fracture Mechanics 75 (16) (2008) 4642-4665.

[19] L. Skarźyński, E. Syroka, J. Tejchman, Measurements and calculations of the width of the fracture process zones on the surface of notched concrete beams, Strain 47 (2011) e319-e332.

[20] A. Carpiuc, Innovative tests for characterizing mixed-mode fracture of concrete : from pre-defined to interactive and hybrid tests, Phd thesis, Uni- 
[21] A. Carpiuc, M. Poncelet, K. Kazimirenko, H. Leclerc, F. Hild, A complex mixed-mode crack propagation test performed with a 6 -axis testing machine and full-field measurements, Engineering Fracture Mechanics (DOI:

[22] M. Ferreira, W. Venturini, F. Hild, On the analysis of notched concrete beams: From measurement with digital image correlation to identification with boundary element method of a cohesive model, Engineering Fracture Mechanics 78 (2011) 71-84.

URL https://tel .archives-ouvertes.fr/tel-01272985

$$
\text { 10.1016/j.engfracmech.2017.01.013). }
$$

3] A. Carpiuc, M. Poncelet, J. Réthoré, S. Roux, CARPIUC Benchmark: Crack Advance, Reorientation, Propagation and Initiation Under Complex loadings, in: CFRAC 2017, Fifth International Conference on Computational Modeling of Fracture and Failure of Materials and Structures, Nantes, France, 14-16 June 2017, 2017.

[24] J. L. Flohic, V. Parpoil, S. Bouissou, M. Poncelet, H. Leclerc., A 3D displacement control by digital image correlation for the multiaxial testing of materials with a Stewart platform., Exp. Mech. 54 (2014) 817-828.

[25] M. Ismail, Novel hexapod-based unidirectional testing and FEM analysis of the RNC isolator, Structural Control and Health Monitoring 23 (6) (2015) 894-922.

[26] J. Guo, D. Wang, R. Fan, W. Chen, G. Zhao, Development of a material testing machine with multi-dimensional loading capability, Journal of Advanced Mechanical Design, Systems, and Manufacturing 10 (2) (2016) JAMDSM0017.

[27] M. Sutton, W. Wolters, W. Peters, W. Ranson, S. McNeill, Determination

versité Paris-Saclay (2015). of displacements using an improved digital correlation method, Image and Vision Computing 1 (3) (1983) 133-139. 
[28] F. Hild, S. Roux, Digital image correlation: from displacement measurement to identification of elastic properties-a review, Strain 42 (2) (2006) 69-80.

[29] D. Hayhurst, Creep rupture under multi-axial states of stress, Journal of the Mechanics and Physics of Solids 20 (6) (1972) 381-382.

[30] D. Hayhurst, A biaxial-tension creep-rupture testing machine, The Journal of Strain Analysis for Engineering Design 8 (2) (1973) 119-123.

[31] S. Demmerle, J. Boehler, Optimal design of biaxial tensile cruciform specimens, Journal of the Mechanics and Physics of Solids 41 (1) (1993) 143-181.

[32] F. Darema, Dynamic Data Driven Applications Systems: A New Paradigm for Application Simulations and Measurements, Springer Berlin Heidelberg, Berlin, Heidelberg, 2004, pp. 662-669.

[33] J. Oden, T. Belytschko, J. Fish, T. Hughes, C. Johnson, D. Keyes, A. Laub, L. Petzold, D. Srolovitz, S. Yip, Simulation-based engineering sciences, Final report, NFS (www.nsf.gov/pubs/reports/sbes_final_report.pdf) (2006).

[34] F. Taucer, CASCADE PROJECT : Recent advences and futur need in experimental earthquake engineering http://elsa.jrc.ec.europa.eu/ publications/Report_7_CASCADE_v2.pdf

[35] V. Saouma, M. Sivaselvan, Hybrid Simulation - Theory, implementation and application, Taylor \& Francis, 2008.

[36] D. Wagg, D. Stoten, Substructuring of dynamical systems via the adaptive minimal control synthesis algorithm, Earthquake engineering \& structural dynamics 30 (6) (2001) 865-877.

[37] O. Bursi, D. Wagg, Modern testing techniques for structural systems Dynamics and control, Springer, 2008. 
[38] M. Nakashima, H. Kato, E. Takaoka, Development of Real-time Pseudo dynamic Testing, Earthquake engineering \& structural dynamics 21 (1) (1992) 79-92.

[39] A. Darby, A. Blakeborough, M. Williams, Improved control algorithm for real-time substructure testing, Earthquake engineering \& structural dynamics 30 (3) (2001) 431-448.

[40] R. Jung, P. Benson Shing, Performance evaluation of a real-time pseudodynamic test system, Earthquake Engineering and Structural Dynamics 35 (7) (2006) 789-810.

[41] S. Roux, F. Hild, Stress intensity factor measurements from digital image correlation: post-processing and integrated approaches, International Journal of Fracture 140 (1-4) (2006) 141-157.

705 [42] F. Mathieu, F. Hild, S. Roux, Identification of a crack propagation law by digital image correlation, International Journal of Fatigue 36 (1) (2012) $146-154$.

[43] J. Réthoré, Automatic crack tip detection and stress intensity factors estimation of curved cracks from digital images, International Journal for Numerical Methods in Engineering 103 (7) (2015) 516-534.

[44] M. Williams, On the Stress Distribution at the Base of a Stationary Crack, Journal of Applied Mechanics 24 (1) (1956) 109-114.

[45] S. Roux, J. Réthoré, F. Hild, Digital image correlation and fracture: an advanced technique for estimating stress intensity factors of $2 \mathrm{D}$ and $3 \mathrm{D}$ cracks, Journal of Physics D: Applied Physics 42 (21) (2009) 214004.

[46] X. Liu, Q. Xiao, B. Karihaloo, XFEM for direct evaluation of mixed mode SIFs in homogeneous and bi-materials, International Journal for Numerical Methods in Engineering 59 (8) (2004) 1103-1118. 
[47] S. Geniaut, P. Massin, N. Moës, A stable 3D contact formulation using X-FEM, European Journal of Computational Mechanics 16 (2) (2007) 259275 .

[48] D. Colombo, An implicit geometrical approach to level sets update for 3D non planar X-FEM crack propagation, Computer Methods in Applied Mechanics and Engineering 237-240 (2012) 39-50.

[49] R. Coulter, Implementation of the Pure Pursuit Path Tracking Algorithm, Tech. Rep. CMU-RI-TR-92-01, Robotics Institute, Pittsburgh, PA (January 1992).

[50] F. Erdogan, G. Sih, On the crack extension in plates under plane loading and transverse shear, Journal of basic engineering 85 (4) (1963) 519-525.

[51] M. Amestoy, H. Bui, K. DangVan, Déviation infinitésimale d'une fissure dans une direction arbitraire, Comptes Rendus de l'Académie des Sciences, Paris 289 (Série B) (1979) 99-103.

[52] J. Qian, A. Fatemi, Mixed mode fatigue crack growth: A literature survey, Engineering Fracture Mechanics 55 (6) (1996) 969-990.

[53] J. Glucklich, On crack stability in some fracture tests, Engineering Fracture Mechanics 3 (3) (1971) 333-344.

[54] J. Nakayama, H. Abe, R. Bradt, Crack Stability in the Work-of-Fracture Test: Refractory Applications, Journal of the American Ceramic Society 64 (11) (1981) 671-675.

[55] E. Lorentz, S. Cuvilliez, K. Kazymyrenko, Convergence of a gradient damage model toward a cohesive zone model, Comptes Rendus Mécanique 339 (1) (2011) 20-26.

[56] S. Lee, Y. Song, S. Han, Biaxial behavior of plain concrete of nuclear containment building, Nuclear Engineering and Design 227 (2) (2004) 143153. 
[57] Code-Aster, Analyse des Structures et Thermomécanique pour des Etudes et des Recherches, version 13, EDF R \& D (http://www.code-aster.org/), 2013.

[58] M. Bottoni, Procédure POST ENDO FISS, EDF R \& D (http://www.codeaster.org/doc/v12/fr/man_u/u4/u4.86.01.pdf), 2013.

[59] K. Kazymyrenko, Réalisation de calculs d'endommagement en quasistatique (rupture fragile), EDF $\mathrm{R} \& \mathrm{D}$ (http://www.codeaster.org/doc/v12/fr/man_u/u2/u2.05.06.pdf), 2014.

[60] F. Hild, S. Roux, Comparison of local and global approaches to digital image correlation, Experimental Mechanics 52 (9) (2012) 1503-1519.

[61] M. Grédiac, F. Hild (Eds.), Full-Field Measurements and Identification in Solid Mechanics, ISTE / Wiley, London (UK), 2012.

[62] F. Mathieu, H. Leclerc, F. Hild, S. Roux, Estimation of elastoplastic parameters via weighted FEMU and integrated-DIC, Experimental Mechanics 55 (1) (2015) 105-119.

[63] S. Avril, M. Bonnet, A. Bretelle, M. Grédiac, F. Hild, P. Ienny, F. Latourte, D. Lemosse, S. Pagano, E. Pagnacco, F. Pierron, Overview of identification methods of mechanical parameters based on full-field measurements, Experimental Mechanics 48 (4) (2008) 381-402.

[64] T. Pottier, F. Toussaint, P. Vacher, Contribution of heterogeneous strain field measurements and boundary conditions modelling in inverse identification of material parameters, European Journal of Mechanics-A/Solids 30 (3) (2011) 373-382.

[65] M. Nooru-Mohamed, Mixed-mode fracture of concrete: an experimental approach, Ph.D. thesis, TU Delft, Delft University of Technology (1992).

[66] M. Bertin, F. Hild, S. Roux, Optimization of a cruciform specimen geometry for the identification of constitutive parameters based upon full-field measurements, Strain 52 (2016) 307-323. 
[67] F. Hild, A. Bouterf, L. Chamoin, H. Leclerc, F. Mathieu, J. Neggers, F. Pled, Z. Tomičević, S. Roux, Toward 4D mechanical correlation, Advanced Modeling and Simulation in Engineering Sciences 3 (1) (2016) 1-26.

[68] M. Bertin, F. Hild, S. Roux, F. Mathieu, H. Leclerc, P. Aimedieu, Integrated digital image correlation applied to elastoplastic identification in a biaxial experiment, Jal of Strain Analysis for Engineering Design 51 (2) (2016) 118-131.

[69] J. Dufour, F. Hild, S. Roux, Displacement and Mechanical Properties from Isogeometric Multiview Stereocorrelation, Jal of Strain Analysis for Engineering Design 50 (7) (2015) 470-487. 